\title{
Impacto Hemodinâmico de Manobra de Recrutamento Alveolar em Pacientes Evoluindo com Choque Cardiogênico no Pós-Operatório Imediato de Revascularização do Miocárdio* Hemodynamic Impact of Alveolar Recruitment Maneuver in Patients Evolving with Cardiogenic Shock in the Immediate Postoperative Period of Myocardial Revascularization
}

\author{
Luiz Marcelo Sá Malbouisson, TSA, Marcelo Brito², Maria José Carvalho Carmona, TSA³, José Otávio Costa Auler Jr, TSA
}

\section{RESUMO}

Malbouisson LMS, Brito M, Carmona MJC, Auler Jr JOC — Impacto Hemodinâmico de Manobra de Recrutamento Alveolar em Pacientes Evoluindo com Choque Cardiogênico no Pós-Operatório Imediato de Revascularização do Miocárdio.

JUSTIFICATIVA E OBJETIVOS: Manobras de recrutamento alveolar (MRA) utilizando pressões de $40 \mathrm{cmH}_{2} \mathrm{O}$ nas vias aéreas são efetivas em reverter as atelectasias após revascularização cirúrgica do miocárdio (RM), contudo não existem estudos avaliando o impacto hemodinâmico dessa manobra em pacientes que evoluíram com choque cardiogênico. O objetivo foi testar a tolerância hemodinâmica à MRA em pacientes evoluindo com choque cardiogênico após $R M$.

MÉTODO: Após admissão na UTI e estabilização hemodinâmica, foram estudados dez pacientes hipoxêmicos e em choque cardiogênico após $R M$. Os ajustes ventilatórios foram volume corrente de $8 \mathrm{~mL}^{\mathrm{kg}} \mathrm{kg}^{-1}$ PEEP $5 \mathrm{cmH}_{2} \mathrm{O}$, FR de $12 \mathrm{ipm}$ e $\mathrm{FiO}$, de 0,6. Pressão contínua de $40 \mathrm{cmH}_{2} \mathrm{O}$ foi aplicada nas vias aéreas por 40 segundos em três ciclos. Entre os ciclos, os pacientes foram ventilados por 30 segundos e após o último ciclo a PEEP foi ajustada em 10 $\mathrm{CmH}_{2} \mathrm{O}$. Foram obtidas medidas hemodinâmicas após 1, 10, 30 e 60 minutos da MRA e colhidas amostras sangüíneas arteriais e venosas para mensuração de lactato e gases sangüíneos 10 e 60 minutos após. Dados analisados por meio de ANOVA e teste de Friedman. Valor de $p$ fixado em 0,05.

RESULTADOS: A MRA aumentou a relação $\mathrm{PaO}_{2} / \mathrm{FiO}_{2}$ de 87 para 129,5 após 10 minutos e 120 após 60 minutos $(p<0,05)$ e reduziu o shunt pulmonar de $30 \%$ para $20 \%(p<0,05)$. Não foram detecta-

${ }^{*}$ Recebido do (Received from) Instituto do Coração do Hospital da Clínicas da Faculdade de Medicina da Universidade de São Paulo (HC-FMUSP), São Paulo, SP

1. Doutor em Ciências pela USP; Médico Supervisor da Divisão de Anestesiologia do HC-FMUSP; Coordenador da UTI/RPA da Disciplina de Anestesiologia do Instituto Central do HC-FMUSP; Especialista em Terapia Intensiva - AMIB

2. Médico Estagiário do Serviço de Anestesiologia e Terapia Intensiva do Instituto do Coração (InCor) do HC-FMUSP

3. Professa-Associada da Disciplina de Anestesiologia da FMUSP; Diretora da Divisão de Anestesiologia do Instituto Central do HC-FMUSP

4. Professor Titular da Disciplina de Anestesiologia da FMUSP; Diretor do Serviço de Anestesiologia Instituto do Coração (InCor) do HC-FMUSP

Apresentado (Submitted) em 9 de fevereiro de 2007

Aceito (Accepted) para publicação em 5 de dezembro de 2007

Endereço para correspondência (Correspondence to):

Dr. Luiz Marcelo Sá Malbouisson

Av. Dr. Enéas de Carvalho Aguiar, I - Cerqueira César

05403-900 São Paulo, SP

E-mail:malbouisson@hcnet.usp.br

(C) Sociedade Brasileira de Anestesiologia, 2008 das alterações hemodinâmicas ou no transporte de oxigênio imediatamente ou em até 60 minutos após a MRA.

CONCLUSÕES: Em pacientes que evoluíram com choque cardiogênico após RM e hipoxemia, a MRA melhorou a oxigenação e foi bem tolerada sob o ponto de vista hemodinâmico.

Unitermos: CIRURGIA, Cardíaca: revascularização do miocárdio; COMPLICAÇÕES: atelectasia; colapso pulmonar; VENTILAÇÃO: manobra de recrutamento alveolar, mecânica, pressão positiva no final da expiração.

\section{SUMMARY}

Malbouisson LMS, Brito M, Carmona MJC, Auler Jr JOC - Hemodynamic Impact of Alveolar Recruitment Maneuver in Patients Evolving with Cardiogenic Shock in the Immediate Postoperative Period of Myocardial Revascularization.

BACKGROUND AND OBJECTIVES: Alveolar recruitment maneu$\operatorname{ver}(A R M)$ with pressures of $40 \mathrm{cmH}_{2} \mathrm{O}$ in the airways is effective in the reversal of atelectasis after myocardial revascularization (MR); however, there is a lack of studies evaluating the hemodynamic impact of this maneuver in patients who evolve with cardiogenic shock after MR. The objective of this study was to test the hemodynamic tolerance to ARM in patients who develop cardiogenic shock after MR.

METHODS: Ten hypoxemic patients in cardiogenic shock after MR were evaluated after admission to the ICU and hemodynamic stabilization. Ventilatory adjustments included tidal volume of $8 \mathrm{~mL} . \mathrm{kg}^{-1}$, PEEP $5 \mathrm{cmH}_{2} \mathrm{O}, \mathrm{RR} 12$, and $\mathrm{FiO}_{2}$ 0.6. Continuous pressure of 40 $\mathrm{CmH}_{2} \mathrm{O}$ was applied to the airways for 40 seconds in three cycles. Between cycles, patients were ventilated for 30 seconds, and after the last cycle, PEEP was set at $10 \mathrm{cmH}_{2} \mathrm{O}$. Hemodynamic measurements were obtained 1, 10,30, and 60 minutes after $A R M$, and arterial and venous blood samples were drawn 10 and 60 minutes after the maneuver to determine lactate levels and blood gases. ANOVA and the Friedman test were used to analyze the data. A $p$ of 0.05 was considered significant.

RESULTS: Alveolar recruitment maneuver increased the ratio $\mathrm{PaO}_{2}$ $\mathrm{FiO}_{2}$ from 87 to 129.5 after 10 minutes and to 120 after 60 minutes $(p<0.05)$ and reduced pulmonary shunting from $30 \%$ to $20 \%(p<0.05)$. Hemodynamic changes or changes in oxygen transport immediately after or up to 60 minutes after the maneuver were not detected.

CONCLUSIONS: In patients who evolved to cardiogenic shock and hypoxemia after MR, ARM improved oxygenation and was well tolerated hemodynamically.

Key Words: COMPLICATIONS: atelectasis; pulmonary collapse; SURGERY, Cardiac: myocardial revascularization; VENTILATION: alveolar recruitment maneuver, mechanical, positive end-expiratory pressure. 
IMPACTO HEMODINÂMICO DE MANOBRA DE RECRUTAMENTO ALVEOLAR EM PACIENTES EVOLUINDO COM CHOQUE CARDIOGÊNICO NO PÓS-OPERATÓRIO IMEDIATO DE REVASCULARIZAÇÃO DO MIOCÁRDIO

\section{INTRODUÇÃO}

O desenvolvimento de atelectasias é observado com freqüência em pacientes submetidos a intervenções cirúrgicas cardíacas, sendo a causa mais importante de hipoxemia e shunt após circulação extracorpórea (CEC) ${ }^{1}$. A incidência de atelectasias em pacientes submetidos à intervenção cirúrgica cardíaca com CEC é alta, variando entre $60 \%$ e $90 \%$ em casuísticas relatadas ${ }^{2,3}$. Avaliando tomografias computadorizadas de tórax em 18 pacientes submetidos a troca valvar mitral ou revascularização do miocárdio (RM) com CEC no primeiro dia pós-operatório, Tenling e col. observaram densidades pulmonares dependentes bilaterais em todos os pacientes com exceção de um. As densidades pulmonares correspondiam a uma fração de aproximadamente $20 \%$ do parênquima pulmonar ${ }^{4}$.

Em pacientes que evoluíram com disfunção ventricular grave e choque cardiogênico, a presença de hipoxemia secundária ao colapso pulmonar pode contribuir para aumentar a resistência e pressão da artéria pulmonar, piorando o desempenho do ventrículo direito e perpetuando o estado de baixo débito cardíaco. A associação de hipoperfusão tecidual, determinada pelo baixo débito cardíaco e conteúdo arterial de oxigênio reduzido, secundário à hipoxemia, pode contribuir para o desenvolvimento de lesões orgânicas à distância. Por outro lado, a possibilidade de efeitos deletérios sobre o sistema cardiovascular limita a eficácia do uso de pressão positiva ao final da expiração (PEEP) elevada por períodos prolongados necessários para a reversão das atelectasias nessa população de pacientes. Manobras de recrutamento alveolar com pressões de $40 \mathrm{cmH}_{2} \mathrm{O}$ nas vias aéreas por curtos períodos têm sido descritas como efetivas em reverter virtualmente todo o colapso pulmonar observado após CEC ${ }^{5}$. Contudo, a aplicação de pressões elevadas pode levar a colapso cardiovascular agudo. O objetivo desse estudo foi avaliar o impacto da aplicação de manobra de recrutamento alveolar com pressões elevadas nas vias aéreas sobre a oxigenação e o desempenho hemodinâmico em pacientes evoluindo com disfunção miocárdica grave no período pós-operatório imediato de revascularização do miocárdio.

\section{MÉTODO}

Após aprovação do Comitê de Ética hospitalar e obtenção do consentimento livre e esclarecido assinado pelo familiar responsável, foram estudados dez pacientes que evoluíram com disfunção miocárdica e que necessitavam de suporte inotrópico e/ou circulatório mecânico no período pós-operatório de revascularização do miocárdio eletiva com circulação extracorpórea. Os critérios de inclusão no estudo foram: a) infusão contínua de dobutamina em doses de $20 \mu \mathrm{g} \cdot \mathrm{kg} \cdot \mathrm{min}^{-1}$ e/ou de milrinona $0,5 \mu \mathrm{g} \cdot \mathrm{kg} \cdot \mathrm{min}^{-1}$; b) saturação venosa mista $<75 \%$ após otimização hemodinâmica; c) ausência de hipovolemia definida como pressão de oclusão da artéria pulmonar (POAP) $<15 \mathrm{mmHg}$; e d) hipoxemia grave caracterizada por relação $\mathrm{PaO}_{2} / \mathrm{FiO}_{2}$ inferior a 150 na gasometria arterial colhida no momento da admissão do paciente na unidade de terapia intensiva. Foram excluídos do estudo: pacientes com hipertensão pulmonar grave, com pressão média na artéria pulmonar $>40 \mathrm{mmHg}$; pacientes com suspeita de hipertensão intracraniana ou com síndrome de fuga aérea (pneumotórax e pneumomediastino à radiografia de tórax ou "borbulhamento" pelos drenos torácicos); pacientes com doenças pulmonares necessitando de oxigênio suplementar no período pré-operatório.

Conforme o protocolo institucional, cada paciente recebeu midazolam 0,1 a $0,2 \mathrm{mg} \mathrm{kg}^{-1}$ por via oral como medicação pré-anestésica 30 minutos antes da intervenção cirúrgica. Após admissão no centro cirúrgico, os pacientes foram monitorados com cardioscópio (derivações $D_{\|}$e V5) e oxímetro de pulso utilizando monitor multiparamétrico Siemens modelo SC7000 (Siemens Medical, Berlim, Alemanha). Acesso venoso com cateter de teflon de calibre $16 \mathrm{G}$ foi obtido em cada paciente. A monitoração da pressão arterial média foi obtida por punção da artéria radial direita sob anestesia local, utilizando-se cateter de teflon de calibre 20G. Após a indução anestésica, foi instalado cateter de artéria pulmonar (CCO/SvO $/$ VIPTM TD catheter; Edwards Healthcare Co., Irvine, CA, EUA) por meio de punção da veia jugular interna direita, devido à fração de ejeção reduzida dos pacientes, conforme o protocolo do serviço de Anestesiologia e Terapia Intensiva. Em todos os pacientes, foi instalada circulação extracorpórea com fluxo não-pulsátil sob hipotermia moderada utilizando oxigenador de membrana OXI Master Century (Braile, São José do Rio Preto, SP, Brasil), circuito preenchido com solução de Ringer com lactato e manitol $50 \mathrm{~g}$. Nos pacientes com dificuldade de saída de circulação extracorpórea após maximização do suporte farmacológico, foi instalado balão intra-aórtico de contrapulsação. Ao final do procedimento, os pacientes foram transportados para a unidade de terapia intensiva.

Após admissão na unidade de terapia intensiva, estabilização hemodinâmica e instalação do suporte ventilatório, era colhida gasometria arterial e obtidas medidas hemodinâmicas iniciais. Os parâmetros iniciais utilizados na ventilação mecânica eram freqüência respiratória de 12 ipm, volume corrente de $8 \mathrm{~mL} . \mathrm{kg}^{-1}$ na modalidade ventilação com pressão controlada, tempo inspiratório de $30 \%$ do ciclo respiratório, PEEP de $5 \mathrm{cmH}_{2} \mathrm{O}$ e $\mathrm{FiO}_{2}$ de 0,6 . O ajuste hemodinâmico era feito num primeiro momento por meio de adequação da volemia visando a manter POAP superior a $18 \mathrm{mmHg}$ e, secundariamente, com otimização de fármacos vasoativos $\mathrm{e}$ do suporte circulatório mecânico com objetivo de manter 0 índice cardíaco acima de 2,5 L. $\mathrm{min}^{-1} \cdot \mathrm{m}^{-2}$ e PAM maior que 65 $\mathrm{mmHg}$. Uma vez obtida estabilização hemodinâmica, eram obtidas gasometrias arteriais e venosas, avaliação hemodinâmica completa e era realizada manobra de recrutamento alveolar (MRA) que consistia na aplicação de pressão contínua nas vias aéreas (CPAP) de $40 \mathrm{cmH}_{2} \mathrm{O}$ durante 40 se- 
gundos em três ciclos. Nos intervalos entre cada ciclo era instituída ventilação mecânica com os mesmos ajustes prévios por 30 segundos, com o objetivo de remover o $\mathrm{CO}_{2}$ alveolar. Após o último ciclo, a PEEP era ajustada em 10 $\mathrm{cmH}_{2} \mathrm{O}$. Após a MRA, foi obtida radiografia de tórax para avaliação da presença de pneumotórax ou outras síndromes de fuga aérea. Medidas hemodinâmicas completas foram obtidas por meio da técnica-padrão de termodiluição (injeções de solução glicosada $5 \%$ à temperatura ambiente em triplicata) 1, 10, 30 e 60 minutos após a MRA. Gasometrias arteriais e venosas foram colhidas nos momentos 10 e 60 minutos após a MRA.

A distribuição normal dos dados hemodinâmicos e gasométricos colhidos foi testada com a utilização do teste de Kolmogorov-Smirnov. Os dados foram analisados por meio de Análise de Variância de uma via para medidas repetidas ou por meio do teste de Friedman, seguidos do teste de múltiplas comparações (Student-Neumann-Keuls ou Wilcoxon), conforme a distribuição dos dados. O nível de significância foi fixado em 0,05 . Os dados estão apresentados como média \pm erro-padrão, ou discriminados se apresentados de forma diferente.

\section{RESULTADOS}

Após admissão e estabilização hemodinâmica inicial dos pacientes na unidade de terapia intensiva por um período médio de 2 horas, 10 pacientes que se mantinham com hipoxemia grave foram estudados. A tabela I mostra os dados hemodinâmicos, de oxigenação e as necessidades de suporte circulatório farmacológico e mecânico dos pacientes imediatamente antes da realização da manobra de recrutamento alveolar. Os pacientes apresentavam índice cardíaco médio de 2,6 L. $\mathrm{min}^{-1} \cdot \mathrm{m}^{2}, \mathrm{SvO}_{2}$ de 64\% (valor normal: $75 \%$ ), extração de oxigênio de $34 \%$ (valor normal: $25 \%$ ) e lactato

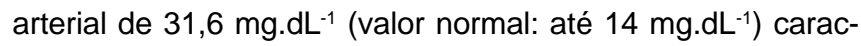
terizando choque cardiogênico. Todos receberam infusão de dobutamina em doses de $20 \mu \mathrm{g} \cdot \mathrm{kg}^{-1} \cdot \mathrm{min}^{-1}$, sete recebiam infusão de milrinona em doses até $0,7 \mu \mathrm{g} \cdot \mathrm{kg}^{-1} \cdot \mathrm{min}^{-1} \mathrm{e}$ oito recebiam infusão de noradrenalina $\left(0,1 \pm 0,03 \mu \mathrm{g} \cdot \mathrm{kg}^{-1} \cdot \mathrm{min}^{-1}\right)$. Cinco dos pacientes necessitaram de assistência circulatória mecânica com balão intra-aórtico de contrapulsação devido à falência do uso isolado de suportes farmacológicos inotrópico e vasopressor. Como pode ser observado, após otimização inicial, a saturação venosa mista média era de $64 \%$, extração de oxigênio de $34 \%$ e lactato arterial de 31,6 $\mathrm{mg} \cdot \mathrm{dL}^{-1}$. Nos pacientes selecionados para participar do estudo, a relação $\mathrm{PaO}_{2} / \mathrm{FiO}_{2}$ mediana, logo após a estabilização hemodinâmica, foi de 87 e a complacência estática foi

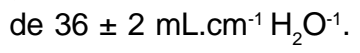

Não foram detectados pneumotórax ou sinais de fuga aérea em radiografia de tórax obtida após a manobra. Como
Tabela I - Dados Antropométricos, Hemodinâmicos e Respiratórios após a Inclusão no Estudo

\begin{tabular}{|c|c|}
\hline Peso $(\mathrm{kg})^{*}$ & $73,2 \pm 3,9$ \\
\hline Altura $(\mathrm{cm})^{*}$ & $50,5 \pm 24,9$ \\
\hline Idade $\left(\right.$ anos) ${ }^{*}$ & $73 \pm 6$ \\
\hline Relação $\mathrm{PaO}_{2} / \mathrm{FiO}_{2}$ & $87,0(69-122)$ \\
\hline Shunt pulmonar* & $30,2 \pm 4,4$ \\
\hline Complacência estática $\left(\mathrm{mL} \mathrm{cmH}_{2} \mathrm{O}^{-1}\right)^{*}$ & $36 \pm 2$ \\
\hline PAM $(\mathrm{mmHg})^{\star}$ & $70,6 \pm 3,1$ \\
\hline $\operatorname{POAP}(\mathrm{mmHg})^{*}$ & $19,6 \pm 1,3$ \\
\hline Índice cardíaco $\left(L \cdot \min ^{-1} \cdot \mathrm{m}^{2}\right)^{*}$ & $2,6 \pm 0,2$ \\
\hline RVS (dinas.seg. $\left.\mathrm{cm}^{-5}\right)^{*}$ & $1625 \pm 132$ \\
\hline ITVSE $\left(\mathrm{gm}^{-1} \cdot \mathrm{m}^{-2} \cdot \text { batimento }\right)^{*}$ & $19,3 \pm 2,0$ \\
\hline Extração de oxigênio (\%)* & $34 \pm 3$ \\
\hline Saturação venosa mista $(\%)^{*}$ & $64,1 \pm 2,2$ \\
\hline Lactato arterial $\left(\mathrm{mg} \cdot \mathrm{dL}^{-1}\right)^{*}$ & $31,6 \pm 17,5$ \\
\hline Dose de dobutamina $\left(\mu \mathrm{g} \cdot \mathrm{kg}^{-1} \cdot \mathrm{min} ; \mathrm{n}=10\right)$ & 20 \\
\hline Dose de milrinona $\left(\mu \mathrm{g} \cdot \mathrm{kg}^{-1} \cdot \min ; \mathrm{n}=7\right)^{*}$ & $0,6 \pm 0,005$ \\
\hline Dose de noradrenalina $\left(\mu \mathrm{g} \cdot \mathrm{kg}^{-1} \cdot \min ; \mathrm{n}=8\right)^{*}$ & $0,1 \pm 0,03$ \\
\hline Assistência circulatória mecânica (n) & 5 \\
\hline
\end{tabular}

* Valores expressos em Média \pm Erro-Padrão.

PAM - pressão arterial média; POAP - pressão de oclusão da artéria pulmonar; RVS - resistência vascular sistêmica; ITSVE índice de trabalho sistólico do ventrículo esquerdo.

pode ser observado no painel superior da figura 1, houve significativo aumento dos valores medianos da relação $\mathrm{PaO}_{2} / \mathrm{FiO}_{2}$ para 129,5 após 10 minutos da MRA, valores que se mantiveram em 120 após 1 hora, enquanto houve redução no shunt pulmonar de $30 \%$ para $20 \%$ após 10 minutos da MRA e que se manteve em $23 \%$ após 60 minutos. Não foram observadas variações hemodinâmicas significativas imediatamente e até 60 minutos após a realização da MRA. O índice cardíaco e a PAM mantiveram-se em níveis estáveis em todos os momentos do estudo (painel inferior - Figura 1). Não foram observadas alterações significativas na pressão de artéria pulmonar, pressão de oclusão da artéria pulmonar, pressão venosa central, freqüência cardíaca e nos índices de trabalho dos ventrículos esquerdo e direito (Figura 2), assim como nas resistências vascular pulmonar e sistêmica à manobra de recrutamento alveolar (painel inferior - Figura 3). Não houve alterações significativas, do ponto de vista estatístico, nas variáveis de transporte de oxigênio (painel superior - Figura 3). 

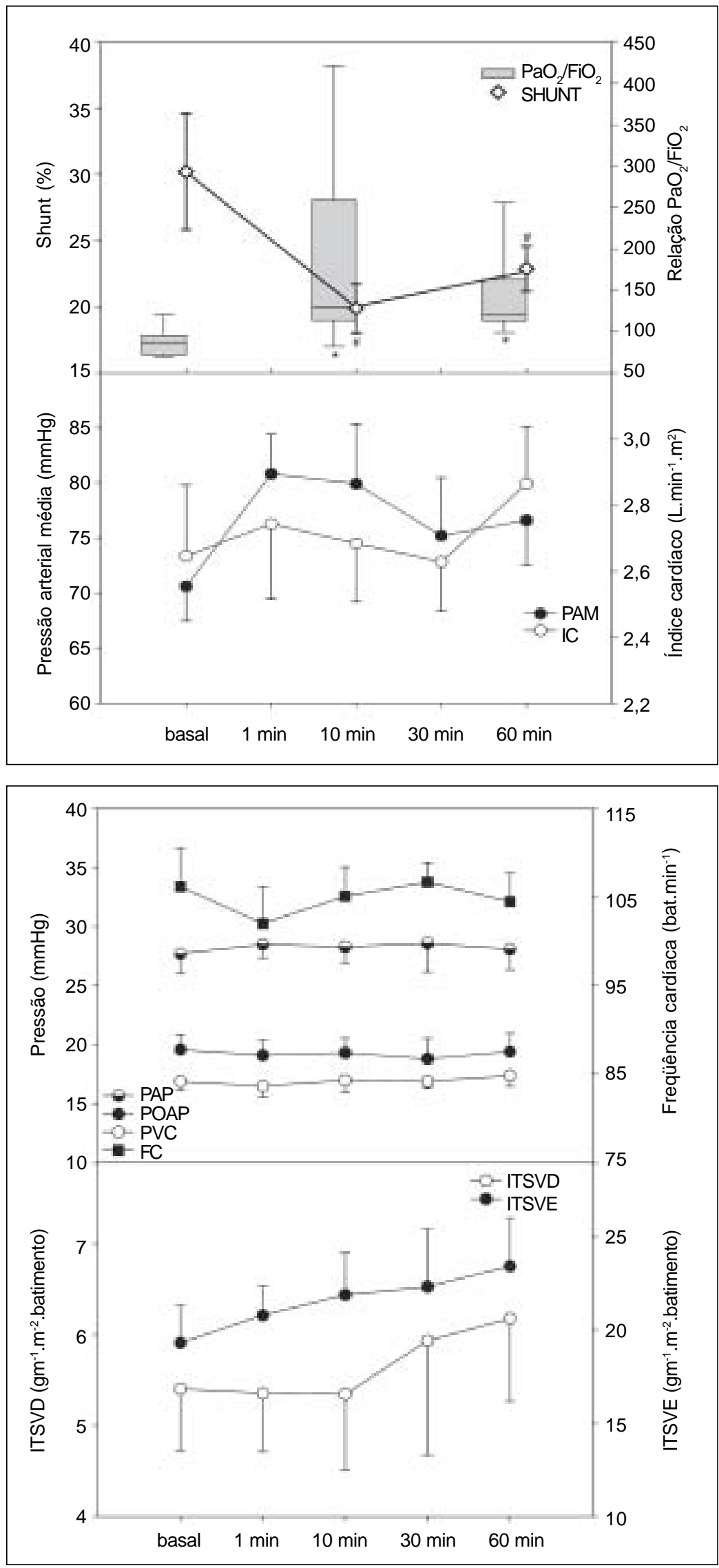

Figura 1 - O painel superior mostra o comportamento do Shunt Pulmonar (diamantes abertos) e da relação $\mathrm{PaO}_{2} / \mathrm{FiO}_{2}$ (barras cinzas) ao longo do tempo após manobra de recrutamento alveolar. $\mathrm{O}$ painel inferior mostra o comportamento temporal da pressão arterial média (PAM) (círculos fechados) e do índice cardíaco (IC) (círculos abertos) ao longo do tempo após manobra de recrutamento alveolar. Não foram observadas alterações significativas nas variáveis hemodinâmicas.

(*) relação $\mathrm{PaO}_{2} / \mathrm{FiO}_{2}$ diferente dos valores basais.

(\#) shunt pulmonar diferente dos valores basais.

Figura 2 - O painel superior mostra o comportamento temporal da Pressão de Artéria Pulmonar (PAP) (círculos semifechados), Pressão Venosa Central (PVC) (círculos abertos), Pressão de Oclusão da Artéria Pulmonar (POAP) (círculos fechados) e da Freqüência Cardíaca (FC) (quadrados fechados) ao longo do tempo após manobra de recrutamento alveolar. O painel inferior mostra o comportamento temporal dos índices de trabalho sistólico de ventrículo esquerdo (ITSVE) (círculos fechados) e do ventrículo direito (ITSVD) (círculos abertos) ao longo do tempo após manobra de recrutamento alveolar. Não foram observadas alterações significativas nas variáveis hemodinâmicas. 


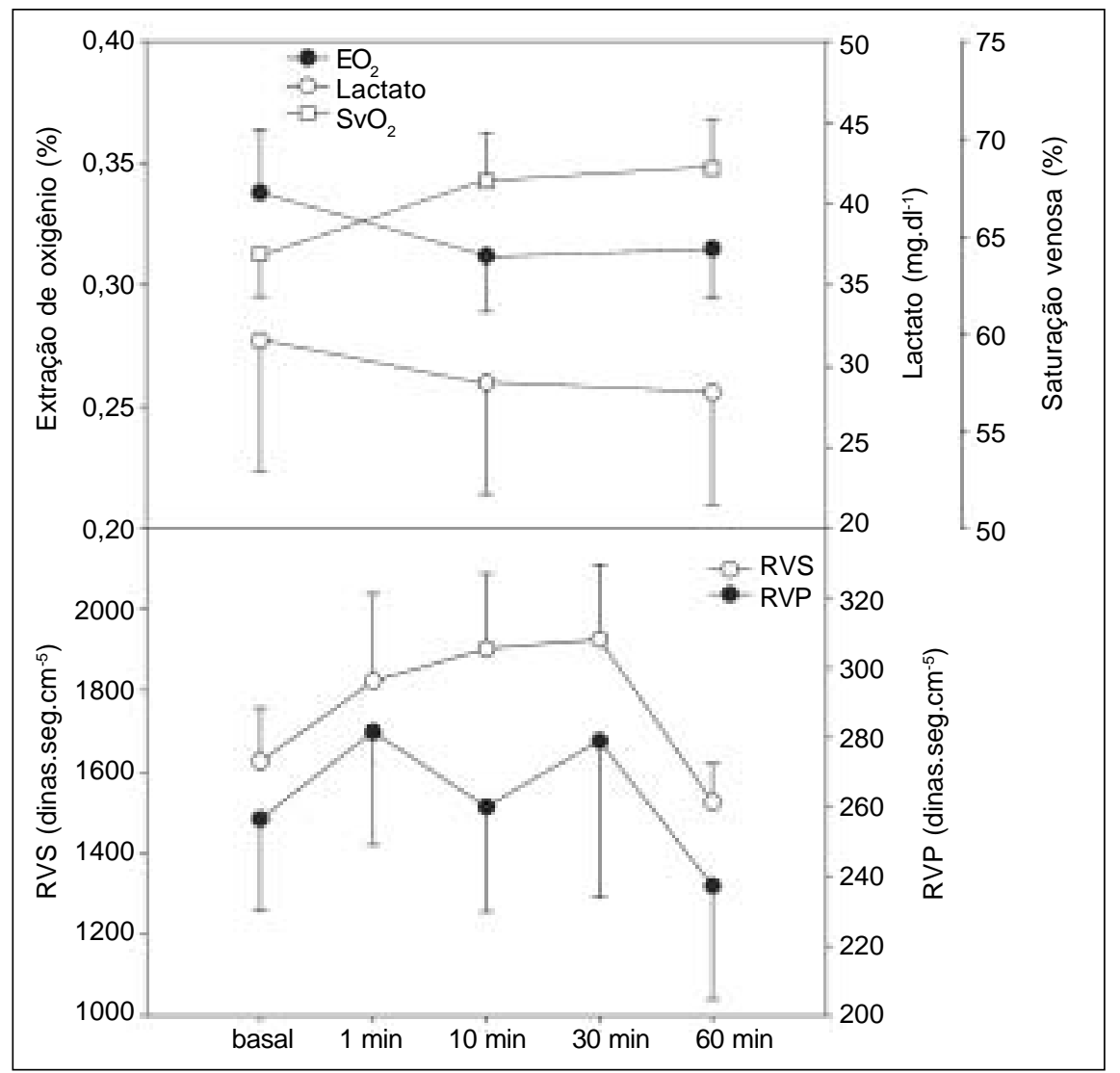

Figura 3 - O painel superior mostra o comportamento temporal do Lactato Arterial (círculos abertos), Taxa de Extração de Oxigênio (círculos fechados) e Saturação Venosa Mista (quadrados abertos) ao longo do tempo. O painel inferior mostra o comportamento temporal da resistência vascular pulmonar (RVP) (círculos fechados) e resistência vascular sistêmica (RVS) (círculos aberto) ao longo do tempo. Não foram observadas alterações significativas nas variáveis estudadas.

\section{DISCUSSÃO}

Neste estudo, foi observado que em pacientes evoluindo com choque cardiogênico no período pós-operatório imediato de revascularização do miocárdio a aplicação de manobra de recrutamento alveolar com pressões nas vias aéreas de $40 \mathrm{cmH}_{2} \mathrm{O}$ promoveu melhora da oxigenação sem induzir alterações significativas no desempenho hemodinâmico imediatamente ou em até 60 minutos após.

O colapso alveolar, seguido de hipoxemia, é uma complicação freqüente nos pacientes submetidos à intervenção cirúrgica cardíaca ${ }^{6}$. As áreas de atelectasia aparecem principalmente nas regiões pulmonares dependentes e caudais, nos primeiros momentos após a infusão de fármacos anestésicos e relaxamento da musculatura, podendo acometer até $20 \%$ de todo o parênquima pulmonar, em até 5 minutos após indução anestésica ${ }^{4,7}$. Nos pacientes submetidos à intervenção cirúrgica cardíaca com circulação extracorpórea, o aumento do volume de água extravascular pulmonar e alteração na atividade normal do sistema surfactante, secundários à ativação das cascatas inflamatória e da coagulação pelo contato do sangue com superfícies não-endotelizadas, contribuem para o aumento do peso do parênquima pulmonar e colapso adicional de unidades alveolares, diminuindo ainda mais a eficiência das trocas gasosas ${ }^{8-10}$. Em pacientes hipoxêmicos com insta- bilidade hemodinâmica, o uso de fração inspirada de oxigênio elevada para compensar a intolerância hemodinâmica à PEEP também pode contribuir para a formação de atelectasias ${ }^{11}$. Neste estudo, foi observado shunt pulmonar médio de $30 \%$ após a estabilização hemodinâmica dos pacientes na unidade de terapia intensiva e relação $\mathrm{PaO}_{2} / \mathrm{FiO}_{2}$ mediana de 87 , configurando situação de lesão pulmonar aguda grave.

A aplicação de manobra de recrutamento alveolar resultou em aumento significativo dos valores medianos da relação $\mathrm{PaO}_{2} / \mathrm{FiO}_{2}$ em $48 \%$, que se mantiveram por pelo menos 60 minutos e redução significativa e duradoura do shunt pulmonar em $10 \%$, refletindo abertura parcial de territórios pulmonares colapsados. Estudos em animais de experimentação e em seres humanos com funções pulmonar e cardiovascular normais submetidos à circulação extracorpórea sob anestesia geral mostram que pressões de $40 \mathrm{cmH}_{2} \mathrm{O}$ são efetivas em re-expandir virtualmente todas as zonas de colapso pulmonar ${ }^{5,12,13}$, assim como PEEP de $10 \mathrm{cmH}_{2} \mathrm{O}$ em prevenir o novo colapso pulmonar ${ }^{14}$. Apesar de evidências de melhora observada nas trocas gasosas após a aplicação de MRA e implementação da PEEP em $10 \mathrm{cmH}_{2} \mathrm{O}$, provavelmente algumas regiões de parênquima pulmonar persistiram colapsadas e incapazes de realizar trocas gasosas adequadas, uma vez que a relação $\mathrm{PaO}_{2} / \mathrm{FiO}_{2}$ manteve-se inferior a 200 e o shunt pulmonar estava em torno 
de $20 \%$, sugerindo presença de desacoplamento residual entre ventilação e perfusão. Nesse grupo de pacientes evoluindo com choque cardiogênico, edema alveolar ${ }^{15}$, alteração da vasorregulação pulmonar induzida pela CEC ${ }^{16}$ ou uso de pressões de vias aéreas aquém das pressões necessárias para abrir e/ou manter abertos os territórios pulmonares previamente colapsados podem explicar a presença de hipoxemia residual. Provavelmente, a repetição da manobra de recrutamento alveolar seguida de aplicação de PEEP em valores mais elevados talvez promovesse meIhoras suplementares da oxigenação.

Do ponto de vista mecânico, a pressão aplicada nas vias aéreas para reverter o colapso pulmonar deve ser superior às pressões que determinam a formação de atelectasias. Em outras palavras, a pressão alveolar tem de ser superior ao somatório das componentes responsáveis pelo aumento da pressão pleural, nominalmente, a pressão imposta pelo peso do parênquima pulmonar edemaciado ${ }^{10,17}$, a compressão dos lobos inferiores induzida pelo peso do coração ${ }^{18}$ e a compressão das regiões caudais determinada pelo peso das vísceras abdominais ${ }^{19}$. A aplicação de pressões de $40 \mathrm{cmH}_{2} \mathrm{O}$ nas vias aéreas, necessárias para reverter as atelectasias, pode, contudo, levar a colapso hemodinâmico, sobretudo em pacientes evoluindo com disfunção miocárdica grave após circulação extracorpórea, sendo evitadas na prática clínica. Neste estudo, apesar de os pacientes estarem evoluindo em choque com sinais de hipoperfusão tecidual, caracterizado por índice cardíaco médio de 2,6 L.min-1. $\mathrm{m}^{2}$ em vigência de suporte circulatório farmacológico maximizado e mecânico, $\mathrm{SvO}_{2}$ de $64 \%$ (valor normal: $75 \%$ ), extração de oxigênio de $34 \%$ (valor normal: $25 \%$ ) e lactato arterial de $31,6 \mathrm{mg}^{-\mathrm{dL}^{-1}}$ (valor normal: até 14 $\mathrm{mg} \cdot \mathrm{dL}^{-1}$ ), a aplicação da MRA foi bem tolerada. Como pode ser observado nas figuras, o comportamento hemodinâmico manteve-se estável, imediatamente e até 60 minutos após a realização da MRA, sem deterioração dos índices de contratilidade, aumento da resistência vascular pulmonar ou piora na oferta tecidual de oxigênio.

Elevações importantes na pressão intratorácica promovem redução no retorno venoso, redução do volume de sangue intratorácico e aumento da impedância à ejeção do ventrículo direito, independentes da compressão direta do coração na fossa cardíaca causada pela acentuada expansão pulmonar ${ }^{20}$. As conseqüências instantâneas da aplicação de manobra de recrutamento alveolar são diminuições abruptas dos volumes sangüíneos dos ventrículos direito e esquerdo, aumento da resistência vascular pulmonar e redução secundária do débito cardíaco por causa da redução do retorno venoso e do volume de sangue nas câmaras cardíacas. A tolerância hemodinâmica à MRA observada nos pa- cientes em choque circulatório poderia ser explicada, em parte, por redução da transmissão das pressões aplicadas nas vias aéreas para o sistema cardiovascular, devido à baixa da complacência do parênquima pulmonar. Teboul e col. ${ }^{21}$ demonstraram relação proporcional entre o impacto causado pela elevação nas pressões de vias aéreas sobre as pressões de enchimento cardíaco e a complacência estática do sistema respiratório. A complacência estática média do sistema respiratório foi de $36 \mathrm{~mL} . \mathrm{cmH}_{2} \mathrm{O}^{-1}$, valores muito abaixo do esperado para pacientes com pulmões normais, que estão acima de $100 \mathrm{~mL} . \mathrm{cmH}_{2} \mathrm{O}^{-1}$. Outro mecanismo que explica a tolerância hemodinâmica à MRA é a otimização da volemia, reduzindo a interferência da manobra no retorno venoso e, por conseqüência, no débito cardíaco. Michard e col. ${ }^{22}$ observaram em pacientes, evoluindo em choque séptico e insuficiência respiratória, que a interferência ventilatória sobre o desempenho hemodinâmico era maior em pacientes hipovolêmicos, causando diminuições cíclicas no débito cardíaco. Jellinek e col. ${ }^{23}$ observaram que PVC abaixo de $12 \mathrm{mmHg}$ estava correlacionada com a redução do débito cardíaco quando era realizada hiperinsuflação pulmonar com pressões de $30 \mathrm{cmH}_{2} \mathrm{O}$ nas vias aéreas, enquanto os pacientes com PVC acima de $12 \mathrm{mmHg}$ apresentavam menor interferência no débito cardíaco. Neste estudo, todos os pacientes tiveram suas pressões de enchimento ventricular otimizadas antes da realização da MRA, com POAP média de 19,6 mmHg em vigência de PEEP de $5 \mathrm{cmH}_{2} \mathrm{O}$. Além disso, nos pacientes nos quais o índice cardíaco não atingiu 2,5 L. min ${ }^{-1} \cdot \mathrm{m}^{-2}$ apenas com infusão de volume, a infusão de fármacos vasoativos foi aumentada, sendo atingidos os objetivos hemodinâmicos em todos os pacientes antes da realização da MRA. Provavelmente, a associação entre hemodinâmica otimizada e redução da complacência estática do sistema respiratório permitiu a realização da MRA sem conseqüências hemodinâmicas ${ }^{24}$. De acordo com os resultados deste estudo, a aplicação da manobra de recrutamento alveolar em pacientes evoluindo com choque circulatório após revascularização do miocárdio com CEC promoveu significativa melhora na oxigenação sem causar prejuízos ao desempenho hemodinâmico. Os benefícios da MRA vão além da reversão das atelectasias. Promovendo melhor distribuição da ventilação para as áreas previamente colapsadas, pode-se reduzir a possibilidade de volutrauma, reduzir a resistência vascular pulmonar associada à hipóxia, melhorando o desempenho do ventrículo direito e diminuindo a necessidade de ventilação mecânica no período pós-operatório. Contudo, estudos são necessários para pesar os benefícios da MRA contra o risco de eventos adversos, como pneumotórax e lesão pulmonar inflamatória associada à hiperinsuflação pulmonar sustentada. 


\section{Hemodynamic Impact of Alveolar Recruitment Maneuver in Patients Evolving with Cardiogenic Shock in the Immediate Postoperative Period of Myocardial Revascularization}

Luiz Marcelo Sá Malbouisson, TSA, M.D.; Marcelo Brito, M.D.; Maria José Carvalho Carmona, TSA, M.D.; José Otávio Costa Auler Jr, TSA, M.D.

\section{INTRODUCTION}

The development of atelectasis is commonly seen in patients undergoing cardiac surgery, representing the most important cause of hypoxemia and shunt after cardiopulmonary bypass (CPB) ${ }^{1}$. The incidence of atelectasis in patients undergoing cardiac surgery with CPB is high, varying from $60 \%$ to $90 \%$ in the studies reported in the literature ${ }^{2,3}$. Evaluating chest CT scans of 18 patients on the first postoperative day of mitral valve replacement or myocardial revascularization (MR) with $\mathrm{CPB}$, Tenlig et al. observed the presence of bilateral dependent pulmonary densities in all but one patient. Those pulmonary densities corresponded to approximately $20 \%$ of the lung parenchyma ${ }^{4}$.

In patients who evolved with severe ventricular dysfunction and cardiogenic shock, the presence of hypoxemia secondary to pulmonary collapse can increase pulmonary artery resistance and pressure, impairing right ventricular performance and perpetuating the state of low cardiac output. The association of tissue hypoperfusion secondary to a low cardiac output, and the reduced arterial oxygen content secondary to hypoxemia can contribute to the development of distant organic lesions. On the other hand, the possibility of noxious effects on the cardiovascular system limits the efficacy of elevated positive end-expiratory pressure (PEEP) for the length of time necessary to reverse atelectasis in this population of patients. Alveolar recruitment maneuver with $40 \mathrm{cmH}_{2} \mathrm{O}$ pressure in the airways for brief moments has been described as effective to reverse virtually all pulmonary collapse observed after CPB ${ }^{5}$. However, the use of elevated pressures can cause acute cardiovascular collapse. The objective of this study was to evaluate the impact of alveolar recruitment maneuver with elevated pressures on oxygenation and hemodynamic performance in patients developing severe myocardial dysfunction in the immediate postoperative period of myocardial revascularization.

\section{METHODS}

After approval by the Ethics Committee of the hospital and signing of the informed consent by the family member responsible for the patient, 10 patients who developed myocardial dysfunction and required inotropic support and/ or mechanical circulatory support after elective myocardial revascularization with cardiopulmonary bypass were evaluated. Inclusion criteria were as follows: a) continuous infusion of $20 \mu \mathrm{g} . \mathrm{kg} \cdot \mathrm{min}^{-1}$ of dobutamine and/or $0.5 \mu \mathrm{g} \cdot \mathrm{kg} \cdot \mathrm{min}^{-1}$ of milrinone; b) mixed venous saturation $<75 \%$ after hemodynamic optimization; c) absence of hypovolemia, defined as pulmonary capillary wedge pressure (PCWP) $<15 \mathrm{mmHg}$; and e) severe hypoxemia, characterized by a $\mathrm{PaO}_{2} / \mathrm{FiO}_{2}$ below 150 in the arterial blood at the time the patient was admitted to the intensive care unit. Patients with severe pulmonary hypertension with mean pulmonary artery pressure $>40 \mathrm{mmHg}$; patients with suspected intracranial hypertension; patients with air-leaking syndrome (pneumothorax and pneumomediastinum on chest X-ray or bubbles draining out of the chest tubes); patients with pulmonary diseases requiring preoperative oxygen supplementation were excluded from the study. According to the protocol of the institution, each patient was pre-medicated with 0.1 to $0.2 \mathrm{mg}^{\mathrm{kg}}{ }^{-1}$ of midazolam PO 30 minutes before the surgery. After arrival to the operating room, patients were monitored with cardioscope $\left(D_{\|}\right.$and V5 derivations) and pulse oximetry using a multiparametric Siemens monitor model SC7000 (Siemens Medical, Berlin, Germany). A 16G Teflon ${ }^{\circledast}$ catheter was used for the peripheral venipuncture. The right radial artery was punctured under local anesthesia with a 20G Teflon $^{\circledR}$ catheter to monitor the mean arterial pressure. After anesthetic induction, a pulmonary artery catheter was introduced (CCO/SvO2/VIPTM TD catheter; Edwards Healthcare Co., Irvine, CA, USA) in the right jugular vein, due to the reduced ejection fraction of the patients, according to the protocol of the Anesthesiology and Intensive Care Department. Non-pulsatile flow cardiopulmonary bypass under moderate hypothermia, with an oxygenator membrane OXI Master Century (Braile, São José do Rio Preto, SP, Brazil), was applied to all patients with the circuit filled with Ringer's lactate and $50 \mathrm{~g}$ of mannitol. Intraaortic balloon counterpulsation was used in patients difficult to wean off cardiopulmonary bypass after maximizing the pharmacological support. At the end of the procedure, patients were transferred to the intensive care unit.

After admission to the intensive care unit, hemodynamic stabilization and ventilatory support, arterial blood gases and initial hemodynamic parameters were obtained. Initial ventilatory parameters included pressure-controlled mode, respiratory rate of $12 \mathrm{bpm}$, tidal volume $8 \mathrm{~mL} . \mathrm{kg}^{-1}$, inspiratory time $30 \%$ of the respiratory cycle, PEEP $5 \mathrm{cmH}_{2} \mathrm{O}$, and $\mathrm{FiO}_{2}$ 0.6. Hemodynamic adjustment was done initially by optimizing the blood volume aiming at maintaining PCWP above 18 $\mathrm{mmHg}$ and by the administration of vasoactive drugs and use of mechanical circulatory support to keep the cardiac index above 2.5 L. $\mathrm{min}^{-1} \cdot \mathrm{m}^{-2}$ and MAP above $65 \mathrm{mmHg}$. When the patient was hemodynamically stable, arterial and venous blood gases were obtained, a complete hemodynamic evaluation was undertaken and the alveolar recruitment maneuver (ARM) consisting of applying continuous $40-\mathrm{cmH}_{2} \mathrm{O}$ 


\section{HEMODYNAMIC IMPACT OF ALVEOLAR RECRUITMENT MANEUVER IN PATIENTS EVOLVING WITH CARDIOGENIC SHOCK}

IN THE IMMEDIATE POSTOPERATIVE PERIOD OF MYOCARDIAL REVASCULARIZATION

pressure to the airways (CPAP) during 40 seconds for three cycles was performed. During the interval between each cycle, mechanical ventilation was resumed for 30 seconds with the same initial parameters to remove alveolar $\mathrm{CO} 2$. After the last cycle, PEEP was adjusted to $10 \mathrm{cmH}_{2} \mathrm{O}$. After ARM, a chest $X$-ray was obtained to evaluate the presence of pneumothorax or other air-leaking syndromes. Complete hemodynamic measurements were obtained by the thermodilution technique (3 injections of D5W at room temperature) 1, 10, 30 , and 60 minutes after the ARM.

The Kolmogorov-Smirnov test was used to evaluate the normal distribution of the hemodynamic parameters and blood gases. The one-way Analysis of Variance for repeated measurements or the Friedman test, followed by the multiple comparison test (Student-Neumann-Keuls or Wilcoxon test) was used to analyze the data according to their distribution. It was considered a level of significance of 0.05 . Data are presented as mean \pm standard error or discriminated if presented in a different form.

\section{RESULTS}

After admission to the intensive care unit and initial hemodynamic stabilization for a mean period of two hours, 10 patients who remained severely hypoxemic were enrolled in the study. Table I shows hemodynamic and oxygenation parameters and the need of pharmacological and mechanical circulatory support of patients immediately before the alveolar recruitment maneuver. Patients had a mean cardiac index of 2.6 L. $\mathrm{min}^{-1} \cdot \mathrm{m}^{2}, \mathrm{SvO}_{2} 64 \%$ (normal: $75 \%$ ), oxygen extraction $34 \%$ (normal: $25 \%$ ), and arterial lactate $31.6{\mathrm{md} . \mathrm{dL}^{-1}}^{-1}$ (normal: up to $14 \mathrm{mg} \cdot \mathrm{dL}^{-1}$ ), characterizing cardiogenic shock. All patients received infusion of $20 \mu \mathrm{g} \cdot \mathrm{kg}^{-1} \cdot \mathrm{min}^{-1}$ of dobutamine, seven received infusion of up to $0.7 \mu \mathrm{g} \cdot \mathrm{kg}^{-1} \cdot \mathrm{min}^{-1}$ of milrinone, and eight received infusion of noradrenaline $(0.1 \pm$ $\left.0.03 \mu \mathrm{g} \cdot \mathrm{kg}^{-1} \cdot \mathrm{min}^{-1}\right)$. Five patients required mechanical circulatory support with intraaortic balloon counterpulsation due to failure of inotropic and vasopressor pharmacologic support. After the initial optimization, mean mixed venous saturation was $64 \%$, oxygen extraction $34 \%$, and arterial lactate $31.6 \mathrm{mg} \cdot \mathrm{dL}^{-1}$. In patients selected for this study, mean $\mathrm{PaO}_{2} / \mathrm{FiO}_{2}$ immediately after hemodynamic stabilization was 87 and static complacency $36 \pm 2 \mathrm{~mL} \mathrm{~cm}^{-1} \mathrm{H}_{2} \mathrm{O}^{-1}$.

Pneumothorax or other signs of air leakage were not detected on the chest X-ray done after the maneuver. As can be seen in the upper panel of Figure 1, there was a significant increase in the mean values of $\mathrm{PaO}_{2} / \mathrm{FiO}_{2}$ to $129.510 \mathrm{mi}-$
Table I - Anthropometric, Hemodynamic, and Respiratory Data After Enrollment in the Study.

\begin{tabular}{|c|c|}
\hline Weight $(\mathrm{kg})^{*}$ & $73.2 \pm 3.9$ \\
\hline Height $(\mathrm{cm})^{*}$ & $50.5 \pm 24.9$ \\
\hline Age $\left(\right.$ years) ${ }^{*}$ & $73 \pm 6$ \\
\hline $\mathrm{PaO}_{2} / \mathrm{FiO}_{2}$ & $87.0(69-122)$ \\
\hline Pulmonary shunt ${ }^{*}$ & $30.2 \pm 4.4$ \\
\hline Static compliance $\left(\mathrm{mL} \cdot \mathrm{cmH}_{2} \mathrm{O}^{-1}\right)^{*}$ & $36 \pm 2$ \\
\hline $\operatorname{MAP}(\mathrm{mmHg})^{*}$ & $70.6 \pm 3.1$ \\
\hline PCWP $(\mathrm{mmHg})^{*}$ & $19.6 \pm 1.3$ \\
\hline Cardiac index $\left(\text { L. } \min ^{-1} \cdot \mathrm{m}^{2}\right)^{*}$ & $2.6 \pm 0,2$ \\
\hline SVR (dinas.seg. $\left.\mathrm{cm}^{-5}\right)^{*}$ & $1625 \pm 132$ \\
\hline LVSWI $\left(\mathrm{gm}^{-1} \cdot \mathrm{m}^{-2} \cdot \text { beat }\right)^{*}$ & $19.3 \pm 2.0$ \\
\hline Oxygen extraction $(\%)^{*}$ & $34 \pm 3$ \\
\hline Mixed venous saturation $(\%)^{*}$ & $64.1 \pm 2.2$ \\
\hline Arterial lactate $\left(\mathrm{mg} \cdot \mathrm{dL}^{-1}\right)^{*}$ & $31.6 \pm 17.5$ \\
\hline Dose of dobutamine $\left(\mu \mathrm{g} \cdot \mathrm{kg}^{-1} \cdot \mathrm{min} ; \mathrm{n}=10\right)$ & 20 \\
\hline Dose of milrinone $\left(\mu \mathrm{g} \cdot \mathrm{kg}^{-1} \cdot \min ; \mathrm{n}=7\right)^{\star}$ & $0.6 \pm 0.005$ \\
\hline Dose of noradrenaline $\left(\mu \mathrm{g} \cdot \mathrm{kg}^{-1} \cdot \min ; \mathrm{n}=8\right)^{*}$ & $0.1 \pm 0.03$ \\
\hline Mechanical circulatory support (n) & 5 \\
\hline
\end{tabular}

nutes after ARM, which was maintained at 120 one hour after the maneuver; and reduction in pulmonary shunting from $30 \%$ to $20 \%$ ten minutes after ARM, which was maintained at $23 \%$ sixty minutes after the maneuver. Significant hemodynamic changes immediately after and 60 minutes after ARM were not observed. Cardiac index and MAP remained stable at all study moments (Figure 1 - lower panel). Significant changes in pulmonary artery pressure, pulmonary capillary wedge pressure, central venous pressure, heart rate, and left and right ventricular work load indexes (Figure 2) were not observed, and the same is true for systemic and pulmonary vascular resistance (Figure 3 - lower panel). There were no statistically significant changes on oxygen transportation (Figure 3 - upper panel). 

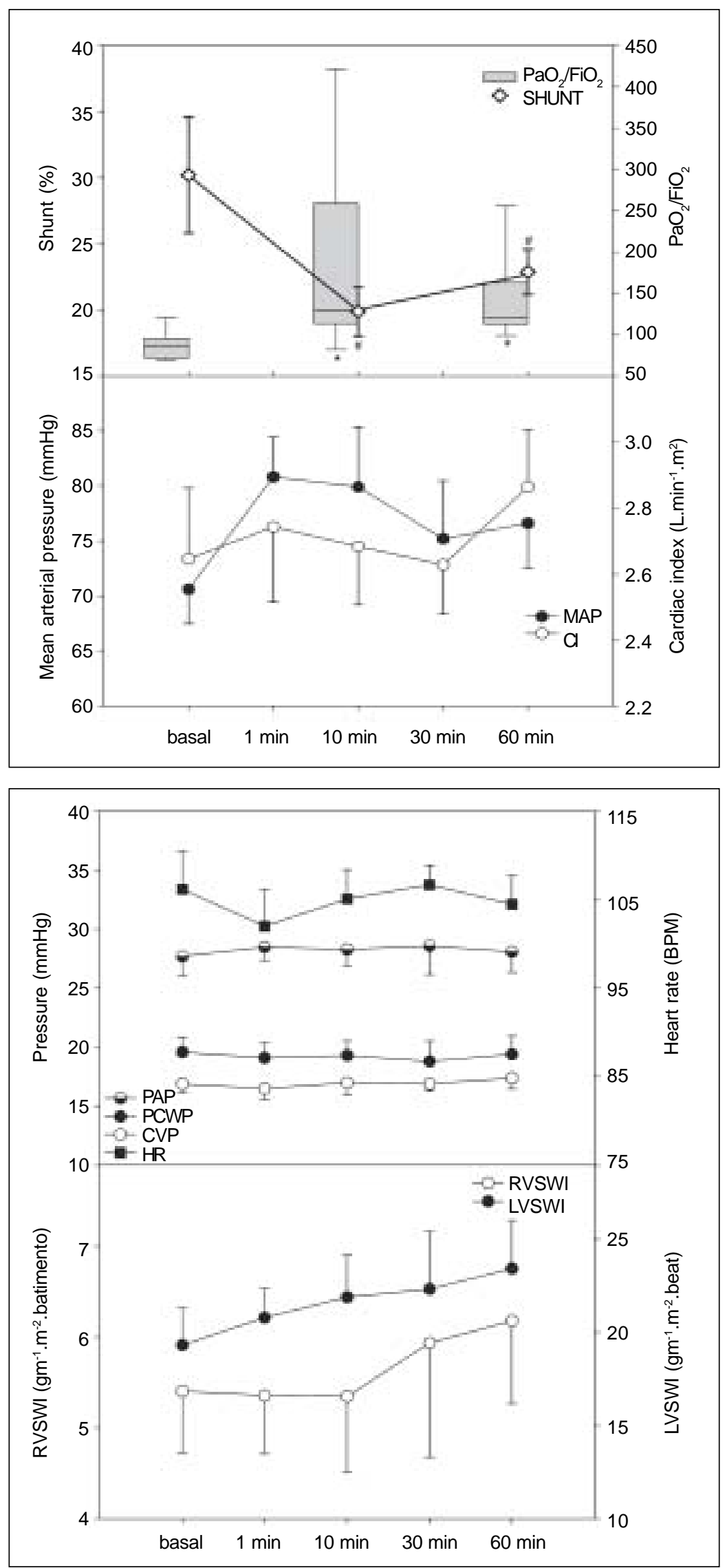

Figure 1 - The upper panel shows the behavior of the Pulmonary Shunt (opened diamonds) and $\mathrm{PaO}_{2} / \mathrm{FiO}_{2}$ (gray bars) along the experiment after alveolar recruiting maneuver. The lower panel shows the temporal behavior of the mean arterial pressure (MAP) (closed circles) and cardiac index (Cl) (opened circles) along the experiment after alveolar recruiting maneuver. Significant hemodynamic changes were not observed.

(*) $\mathrm{PaO}_{2} / \mathrm{FiO}_{2}$ differs from baseline values.

(\#) pulmonary shunt is different from baseline values.
Figure 2 - The upper panel shows the temporal behavior of the Pulmonary Artery Pressure (PAP) (closed semi-circles), Central Venous Pressure (CVP) (opened circles), Pulmonary Capillary Wedge Pressure (PCWP) (closed circles), and Heart Rate (HR) (closed squares) along the experiment after alveolar recruiting maneuver. The lower panel shows the temporal behavior of the left ventricular systolic work index (LVSWI) (closed circles) and right ventricular systolic work index (RVSWI) (opened circles) along the study after alveolar recruiting maneuver. Significant changes in hemodynamic parameters were not observed. 


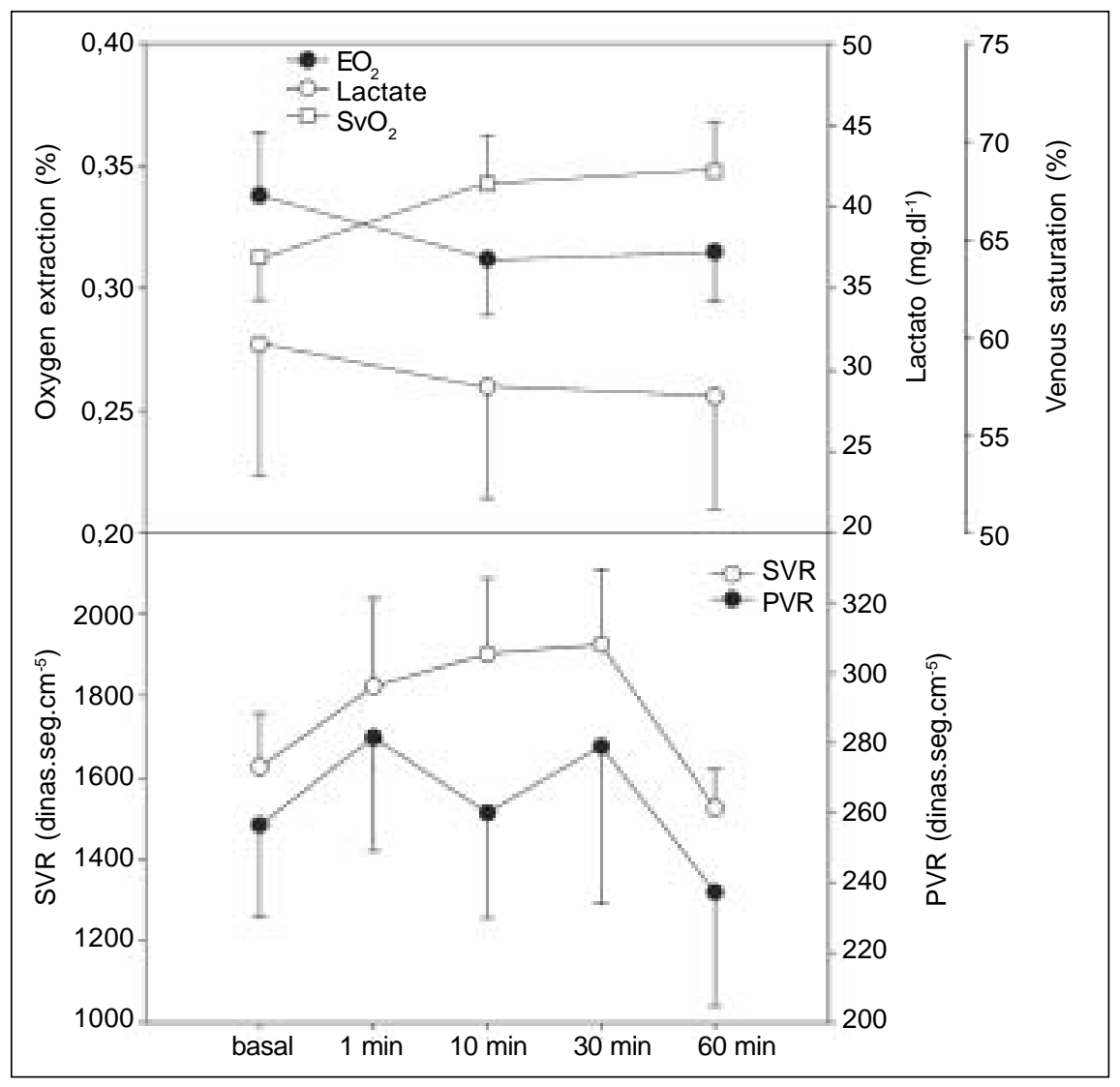

Figure 3 - The upper panel shows the temporal behavior of Arterial Lactate (opened circles), Oxygen Extraction Rate (closed circles), and Mixed Venous saturation (opened squares) along the study. The lower panel shows the temporal behavior of the pulmonary vascular resistance (PVR) (closed circles) and systemic vascular resistance (SVR) (opened circles) along the study. Significant changes in the parameters were not observed.

\section{DISCUSSION}

In this study, we observed that in patients who develop cardiogenic shock in the immediate postoperative period of myocardial revascularization, alveolar recruitment maneuver with airways pressures of $40 \mathrm{cmH}_{2} \mathrm{O}$ improved oxygenation without significant changes in hemodynamic performance, immediately or up to 60 minutes after the maneuver.

Alveolar collapse followed by hypoxemia is a common complication in patients undergoing cardiac surgeries ${ }^{6}$. Atelectasis develop especially in dependent and caudal pulmonary regions during the first moments of anesthetic infusion and muscular relaxation, affecting up to $20 \%$ of the pulmonary parenchyma, up to 5 minutes after anesthetic induction ${ }^{4,7}$. In patients undergoing cardiac surgery with cardiopulmonary bypass, the increase in pulmonary extravascular water and changes in the normal activity of the surfactant system, secondary to the activation of inflammatory and coagulation cascades by the contact between the blood and non-endothelial surfaces, contribute to the increased weight of the pulmonary parenchyma and additional alveolar collapse, further decreasing the efficacy of gas exchange ${ }^{8-10}$. In hypoxemic patients with hemodynamic instability, the use of increased inspired fraction of oxygen to compensate the hemodynamic intolerance to PEEP, can also contribute to the development of atelectasis ${ }^{11}$. In the present study, it was observed a mean pulmonary shunt of $30 \%$ after hemodynamic stabilization in the intensive care unit, and mean $\mathrm{PaO}_{2} / \mathrm{FiO}_{2}$ of 87 , which configure severe acute pulmonary lesion.

Alveolar recruitment maneuver resulted in a significant increase of mean $\mathrm{PaO}_{2} / \mathrm{FiO}_{2}$ values of $48 \%$, which were maintained for at least 60 minutes, and a significant and lasting reduction in pulmonary shunting by $10 \%$ reflecting the partial opening of collapsed lung areas. Experimental animal studies and studies in humans with normal pulmonary and cardiovascular functions undergoing cardiopulmonary bypass under general anesthesia demonstrated that pressures of $40 \mathrm{cmH}_{2} \mathrm{O}$ are effective in re-opening virtually all areas of lung collapse ${ }^{5,12,13}$, as well as the efficacy of a PEEP of $10 \mathrm{cmH}_{2} \mathrm{O}$ in preventing new areas of pulmonary collapse ${ }^{14}$. Despite the evidence of improved gas exchange after ARM and implementation of PEEP of $10 \mathrm{cmH}_{2} \mathrm{O}$, it is most likely that some areas of the pulmonary parenchyma remained collapsed and were unable to provide adequate gas exchange, since $\mathrm{PaO}_{2} / \mathrm{FiO}_{2}$ remained below 200 and pulmonary shunting remained around $20 \%$, suggesting the presence of residual ventilation/perfusion imbalance. In this group of patients who evolved with cardiogenic shock, alveolar edema ${ }^{15}$, changes in pulmonary vascular regulation induced by the CPB ${ }^{16}$, or the use of elevated pressures in the 
airways below those necessary to open and/or maintain opened previously collapsed pulmonary areas can explain the presence of residual hypoxemia. It is possible that repeating the alveolar recruiting maneuver followed by higher PEEP would have lead to supplementary improvement in oxygenation.

The pressure applied on the airways to reverse the pulmonary collapse should be higher than the pressures that caused the atelectasis. In other words, the alveolar pressure has to be higher than the sum of the components responsible for the increase in pleural pressure, namely the pressure imposed by the weight of the edematous lung parenchyma ${ }^{10,17}$, compression of the lower lobes by the weight of the heart ${ }^{18}$, and compression of caudal regions by the weight of the abdominal viscera ${ }^{19}$. However, the use of $40 \mathrm{cmH}_{2} \mathrm{O}$ pressures on the airways, necessary to reverse atelectasis, can cause hemodynamic collapse especially in patients who develop severe myocardial dysfunction after cardiopulmonary bypass and, therefore, it is avoided. In this study, although patients were evolving with shock and had signs of tissue hypoperfusion, characterized by a mean cardiac index of 2.6 L. $\min ^{-1} \cdot \mathrm{m}^{2}$ in the presence of maximal pharmacological and mechanical circulatory support, $\mathrm{SvO}_{2} 64 \%$ (normal: $75 \%$ ), oxygen extraction 34\% (normal: $25 \%$ ), and arterial lactate 31.6 mg.dL-1 (normal: up to $14 \mathrm{mg} \cdot \mathrm{dL}^{-1}$ ), ARM was performed without deterioration of contractility indexes, increase in pulmonary vascular resistance or worsening tissue oxygenation. Important elevations in intra-thoracic pressure cause a reduction in venous return and the volume of intra-thoracic blood, and increase impedance to right ventricular ejection, independent of the direct compression of the heart in the cardiac groove caused by the increased pulmonary expansion ${ }^{20}$. Immediate consequences of the alveolar recruitment maneuver include sudden reduction in right and left ventricular blood volume, increase in pulmonary vascular resistance, and reduction in cardiac output secondary to the reduced venous return and blood volume in the cardiac chambers. The hemodynamic tolerance to ARM observed in patients in circulatory shock can be partly explained by a reduction in the transmission of the airways pressure to the cardiovascular system due to the low pulmonary complacency. Teboul et al. ${ }^{21}$ demonstrated a proportional relationship between the impact caused by an elevation of airways pressure on cardiac filling pressures and static complacency of the respiratory system. The mean static complacency of the respiratory system was $36 \mathrm{~mL} . \mathrm{cmH}_{2} \mathrm{O}^{-1}$, much lower than expected for patients with normal lungs whose pressures are above $100 \mathrm{~mL} \cdot \mathrm{H}_{2} \mathrm{O}^{-1}$. Another mechanism that explains the hemodynamic tolerance to ARM is the optimization of blood volume, which reduces the interference of the maneuver on venous return and, consequently, on cardiac output. Michard et al. ${ }^{22}$ observed, in patients with septic shock and respiratory failure that the ventilatory interference on hemodynamic performance was greater in hypovolemic patients causing cyclic reductions in cardiac output. Jellineck et al. ${ }^{23}$ observed that a CVP below than $12 \mathrm{mmHg}$ was associated with a reduction in cardiac output with pulmonary hyperinsuflation with pressures of $30 \mathrm{cmH}_{2} \mathrm{O}$ in the airways, while this interference was less accentuated in patients with CVP higher than $12 \mathrm{mmHg}$. In the present study, ventricular filling pressures were optimized in all patients before ARM, with a mean PCWP of $19.6 \mathrm{mmHg}$ in face of a PEEP of $5 \mathrm{cmH}_{2} \mathrm{O}$. Besides, in patients whose cardiac index did not reach 2.5 L. $\mathrm{min}^{-1} \cdot \mathrm{m}^{-2}$ just with volume replacement, the infusion of vasoactive drugs was increased and the hemodynamic goals were achieved in all patients before ARM. It is possible that the association of optimized hemodynamic parameters and the reduction in the static complacency of the respiratory system allowed for ARM without hemodynamic consequences ${ }^{24}$.

According to the results of the present study, alveolar recruitment maneuver in patients with circulatory shock after myocardial revascularization with CPB promoted a significant improvement in oxygenation without jeopardizing the hemodynamic performance. The benefits of the ARM go beyond reversion of atelectasis. By promoting better distribution of the ventilation to previously collapsed areas, it reduces the possibility of volumetric trauma, reduces pulmonary vascular resistance associated with hypoxia, improves right ventricular performance and reduces the need for postoperative mechanical ventilation. However, further studies are necessary to compare the benefits of ARM to the risks of adverse events, such as pneumothorax and inflammatory lung damage associated with sustained pulmonary hyperinflation.

\section{REFERÊNCIAS - REFERENCES}

01. Magnusson L, Zemgulis V, Wicky S et al. - Atelectasis is a major cause of hypoxemia and shunt after cardiopulmonary bypass: an experimental study. Anesthesiology, 1997;87:1153-1163.

02. Gale GD, Teasdale SJ, Sanders DE et al. - Pulmonary atelectasis and other respiratory complications after cardiopulmonary bypass and investigation of aetiological factors. Can Anaesth Soc J, 1979;26:15-21.

03. Emhardt JD, Moorthy SS, Brown JW et al. - Chest radiograph changes after cardiopulmonary bypass in children. J Cardiovasc Surg (Torino), 1991;32:314-317.

04. Tenling A, Hachenberg $\mathrm{T}$, Tyden $\mathrm{H}$ et al. - Atelectasis and gas exchange after cardiac surgery. Anesthesiology, 1998;89:371378.

05. Magnusson L, Zemgulis V, Tenling A et al. - Use of a vital capacity maneuver to prevent atelectasis after cardiopulmonary bypass:an experimental study. Anesthesiology, 1998;88:134-142.

06. Hachenberg T, Brussel T, Roos N et al. - Gas exchange impairment and pulmonary densities after cardiac surgery. Acta Anaesthesiol Scand, 1992;36:800-805.

07. Brismar B, Hedenstierna G, Lundquist $\mathrm{H}$ et al. - Pulmonary densities during anesthesia with muscular relaxation: a proposal of atelectasis. Anesthesiology, 1985;62:422-428.

08. Wasowicz M, Sobczynski P, Drwila R et al. - Air-blood barrier injury during cardiac operations with the use of cardiopulmonary bypass (CPB). An old story? A morphological study. Scand Cardiovasc J, 2003;37:216-221. 


\section{HEMODYNAMIC IMPACT OF ALVEOLAR RECRUITMENT MANEUVER IN PATIENTS EVOLVING WITH CARDIOGENIC SHOCK IN THE IMMEDIATE POSTOPERATIVE PERIOD OF MYOCARDIAL REVASCULARIZATION}

09. Griese M, Wilnhammer C, Jansen S et al. - Cardiopulmonary bypass reduces pulmonary surfactant activity in infants. $J$ Thorac Cardiovasc Surg, 1999;118:237-244.

10. Pelosi P, D'andrea L, Vitale G et al. - Vertical gradient of regional lung inflation in adult respiratory distress syndrome. Am J Respir Crit Care Med, 1994;149:8-13.

11. Joyce CJ, Williams $A B$ - Kinetics of absorption atelectasis during anesthesia:a mathematical model. J Appl Physiol, 1999;86: 1116-1125.

12. Rothen HU, Sporre B, Engberg G et al. - Re-expansion of atelectasis during general anaesthesia:a computed tomography study. Br J Anaesth, 1993;71:788-795.

13. Claxton BA, Morgan $\mathrm{P}$, McKeague $\mathrm{H}$ et al. - Alveolar recruitment strategy improves arterial oxygenation after cardiopulmonary bypass. Anaesthesia, 2003;58:111-116.

14. Rothen HU, Sporre B, Engberg G et al. - Prevention of atelectasis during general anaesthesia. Lancet, 1995;345:1387-1391.

15. Anyanwu E, Dittrich $\mathrm{H}$, Gieseking $\mathrm{R}$ et al. - Ultrastructural changes in the human lung following cardiopulmonary bypass. Basic Res Cardiol, 1982;77:309-322.

16. Kirshbom PM, Jacobs MT, Tsui SS et al. - Effects of cardiopulmonary bypass and circulatory arrest on endothelium-dependent vasodilation in the lung. J Thorac Cardiovasc Surg, 1996;111: 1248-1256.

17. Sandiford P, Province MA Schuster DP - Distribution of regional density and vascular permeability in the adult respiratory distress syndrome. Am J Resp Crit Care Med, 1995;151:737-742.

18. Malbouisson LM, Busch CJ, Puybasset $L$ et al. - Role of the heart in the loss of aeration characterizing lower lobes in acute respiratory distress syndrome. CT Scan ARDS Study Group. Am J Respir Crit Care Med, 2000;161:2005-2012.

19. Froese $A B$, Bryan $A C$ - Effects of anesthesia and paralysis on diaphragmatic mechanics in man. Anesthesiology, 1974;41: 242-254.

20. Pinsky MR - Recent advances in the clinical application of heartlung interactions. Curr Opin Crit Care, 2002;8:26-31.

21. Teboul JL, Pinsky MR, Mercat A et al. - Estimating cardiac filling pressure in mechanically ventilated patients with hyperinflation. Crit Care Med, 2000;28:3631-3636.

22. Michard F, Boussat S, Chemla D et al. - Relation between respiratory changes in arterial pulse pressure and fluid responsiveness in septic patients with acute circulatory failure. Am J Respir Crit Care Med, 2000;162:134-138.

23. Jellinek $H$, Krafft $P$, Fitzgerald RD et al. - Right atrial pressure predicts hemodynamic response to apneic positive airway pressure. Crit Care Med, 2000;28:672-678.

24. Auler Jr JOC, Nozawa E, Toma EK et al. - Manobra de recrutamento alveolar na reversão da hipoxemia no pós-operatório imediato em cirurgia cardíaca. Rev Bras Anestesiol, 2007;57: 476-488.

\section{RESUMEN}

Malbouisson LMS, Brito M, Carmona MJC, Auler Jr JOC — Impacto Hemodinámico de Maniobra de Reclutamiento Alveolar en Pacientes Evolucionando con Choque Cardiogénico en el Postoperatorio Inmediato de Revascularización del Miocardio.

JUSTIFICATIVA Y OBJETIVOS: Maniobras de reclutamiento alveolar (MRA) utilizando presiones de $40 \mathrm{cmH}_{2} \mathrm{O}$ en las vías aéreas son efectivas en revertir las atelectasias después de la revascularización quirúrgica del miocardio (RM), sin embargo, no existen estudios que evalúen el impacto hemodinámico de esta maniobra en pacientes que evolucionaron con choque cardiogénico. El objetivo fue probar la tolerancia hemodinámica a la MRA en pacientes que evolucionan con choque cardiogénico después de la RM.

MÉTODO: Después de la entrada en la UCl y de la estabilización hemodinámica, se estudiaron 10 pacientes hipoxémicos y en choque cardiogénico después de RM. Los ajustes de ventilación fueron volumen corriente de $8 \mathrm{~mL} . \mathrm{kg}^{-1}$, PEEP $5 \mathrm{cmH}_{2} \mathrm{O}$, FR de $12 \mathrm{ipm} \mathrm{y}$ $\mathrm{FiO}_{2}$ de 0,6 . Presión continua de $40 \mathrm{cmH}_{2} \mathrm{O}$ se aplicó en las vías aéreas por 40 segundos en tres ciclos. Entre los ciclos, los pacientes fueron ventilados por 30 segundos y después del último ciclo, la PEEP fue ajustada en $10 \mathrm{cmH}_{2} \mathrm{O}$. Fueron obtenidas medidas hemodinámicas después de 1, 10, 30 y 60 minutos de la MRA y recogidas muestras de sangre arteriales y venosas para la medida de lactato y de los gases sanguíneos 10 y 60 minutos después. Datos analizados a través de ANOVA y test de Friedman. Valor de p fijado en 0,05.

RESULTADOS: La MRA aumentó la relación $\mathrm{PaO}_{2} / \mathrm{FiO}_{2}$ de 87 para 129,5 después de 10 minutos y 120 después de 60 minutos $(p<$ $0,05)$ y redujo el shunt pulmonar de $30 \%$ para $20 \%(p<0,05)$. No se detectaron alteraciones hemodinámicas o en el transporte de oxígeno inmediatamente o en hasta 60 minutos después de la MRA.

CONCLUSIONES: En pacientes que evolucionaron con choque cardiogénico después de RM e hipoxemia, la MRA mejoró la oxigenación y fue bien tolerada hemodinámicamente. 